\title{
Hardening description for FCC materials under complex loading paths
}

\author{
C. Gérard ${ }^{\mathrm{a}, \mathrm{b}, \mathrm{c}}$ B. Bacroix ${ }^{\mathrm{c}}$ M. Bornert ${ }^{\mathrm{b}}$ G. Cailletaud ${ }^{\mathrm{a}}$ J. Crépin ${ }^{\mathrm{b}}$ S. Leclercq ${ }^{\mathrm{d}}$ \\ ${ }^{a}$ Centre des Matériaux, Mines Paristech, CNRS-UMR 7633, BP 87, 91003 Evry, France \\ b Laboratoire de Mécanique du Solide, Ecole Polytechnique, CNRS-UMR 7649, 91128 Palaiseau Cedex, France \\ ${ }^{\mathrm{c}}$ Laboratoire des Propriétés Mécaniques et Thermodynamiques des Matériaux, Université Paris 13, CNRS-UPR 9001 , 93430 Villetaneuse, \\ France \\ ${ }^{\mathrm{d}}$ EDF RधBD, Département Matériaux et Mécanique des Composants, Les Renardières, 77818 Moret-sur-Loing Cedex, France
}

\begin{abstract}
The present work aims at exploring self and latent hardening for FCC polycrystals under complex loading paths at room temperature. Combinations of simple loading paths sequences, such as tension and simple shear, with different orientations with regard to rolling direction, are considered. Experimental results are compared to finite element computations of polycrystalline aggregates taking into account the material microstructure, and to simulations based on mean field models.
\end{abstract}

Key words: Self hardening, latent hardening, complex loading paths, finite element, parallel computation, scale transition rules.

\section{Introduction}

The plastic properties of face-centred cubic (FCC) crystals are experimentally well documented. They are related to dislocation arrangement and microstructure formations but little is known experimentally about a quantitative description of the slip system interaction. Only indirect and approximate estimates can be obtained through latent hardening tests on single crystals [6], [18].

Mandel [11] proposed to describe the interaction between slip systems by a matrix separating self and latent hardening. Later, Franciosi [6] defined an interaction matrix to consider the interactions in FCC crystals. The number of distinct interaction coefficients between the twelve mutually interacting slip systems in the FCC structure is reduced to only six for symmetry reasons. In the past, the interac-

Email address: celine.gerard@mat.ensmp.fr (C. Gérard). 
tion matrix has been introduced either in phenomenological models, following for instance Asaro [1], or in dislocationbased models. A phenomenological approach will be used in the present paper [12]. It has already been applied to copper bicrystals [13]. Dislocation densities are classically introduced in "dislocation driven" plasticity models, for instance in [17]. This class of model was also used in our work, but is not presented here due to the lack of space. In fact, the conclusions concerning the interaction matrix are the same with both model types. On the other hand, the nature of the interactions is naturally represented in lower scale approaches, like discrete dislocation dynamics simulation (DDD). The shape of the matrix can be then investigated. Recent results [5] report innovative values of the interaction coefficients, specially for the collinear systems. The motivations of our study is then: to perform discriminating tests using multiaxial loading paths, to trigger system interaction; to calibrate the crystal plasticity model with respect to the global responses ; to check the values used in the interaction matrix with respect to the DDD results.

\section{Methodology}

Method of identification. The identification process uses finite element computations to calibrate the material parameters of the crystal plasticity model, so that the description does not involve any questionnable scale transition rule. Periodic boundary conditions are adopted. As overall stress conditions cannot be imposed on parallel periodic computations in the present implementation, the overall strain tensor has been prescribed. The definition of the transverse loading is a classical issue for this case of problem [8]. In order to simulate uniaxial tension (with vanishing overall stress components except along the tensile direction), an overall strain history induced by uniaxial tension was first determined by using a mean field model described below (the $\beta$-rule). The transverse components obtained in this computation are imposed to the finite element calculation, and iteratively adjusted if necessary, in order to reach a zero transverse stress. In the final computations, the perturbation on the value of the transverse stress components were less than $5 \mathrm{MPa}$, and considered negligible with respect to the tensile stress. Such a procedure ensures that loading conditions in the finite element simulations and the mean field models described hereafter are similar, so that the two types of approaches can be compared.

Material and experimental procedure. Four loading paths are studied: two tension tests and two combinations of shear and tensile loadings. The material used is a $0.5 \mathrm{~mm}$ sheet of hot rolled copper OFHC.

The texture was measured by X-ray diffraction. The diffractometer used is a 4 circle goniometer located at the exit of a monochromatic cobalt beam. Pole figures are corrected of background noise, defocalisation, and then normalised. Texture measurements are realised on the most intensive $h k l$ plane families: $\{111\},\{200\},\{220\}$. Orientations distributions functions are then calculated with the harmonic method. Pole figures are presented in Fig.3. A marked cubic texture is obtained.

The shear direction axis 1 in Fig. 1 is parallel to the rolling direction of the sheet. The prestrain device and the sample geometry (see Fig.1 for dimensions) have already been described in [2], [7]. The displacement is precisely measured by a digital image correlation technique making use of a $2 \mathrm{~mm}$-step grid serigraphied at the surface of the sample. The macroscopic shear strain is $\varepsilon_{12}=1.4 \%$. Smaller tensile specimens were cut out from the large homogeneously 
prestrained sample at two different angles $\alpha$ from the prestrain direction: $0^{\circ}$ and $45^{\circ}$. All specimens were realised by electrical discharge machining. During tensile loading, the displacement is measured by a $14 \mathrm{~mm}$ base extensometer.
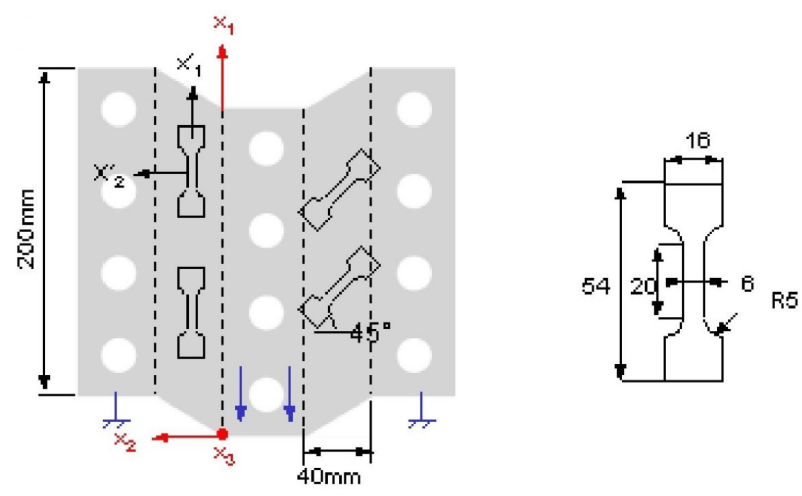

Fig. 1. Geometry of the samples used in the prestrain by simple shear device.

The macroscopic tensile curves (Fig.5) show that the texture effect seems quite negligible (no difference between the tensile tests on specimens at $0^{\circ}$ and $45^{\circ}$ ). On the other hand, over-hardening in tension at $45^{\circ}$ after pre-shear is $11 \mathrm{MPa}$, and the over-hardening of the orthogonal loading is $34 \mathrm{MPa}$. The uncertainty of stress evaluation is about $5 \%$.

Single crystal model. The single crystal constitutive equations are written in the framework of a phenomenological crystal plasticity approach [12] with isotropic hardening on slip systems. The strain rate $\dot{\gamma}^{s}$ on system $s$ is described by a power function:

$$
\dot{\gamma^{s}}=\left\lfloor\frac{\left|\tau^{s}\right|-r^{s}}{K}\right\rfloor^{n} \operatorname{sign}\left(\tau^{s}\right),
$$

where $\lfloor a\rfloor$ is the positive part of $a$, and where $K$ and $n$ are material parameters. The evolution of the isotropic hardening variable $r^{s}$ takes into account slip system interactions by means of an interaction matrix, with components $h_{r s}$ :

$$
r^{s}=r_{0}+Q \sum h^{s r}\left\{1-e^{\left(-b v^{r}\right)}\right\}
$$

where $r_{0}$ is the initial critical resolved shear stress, $Q$ the hardening capacity, $b$ a material coefficient, and $v^{r}$ the accumulated slip on system $r$, such that $\dot{v}^{r}=\left|\dot{\gamma}^{s}\right|$.

The orientation tensor for the system $s, \mathbf{m}^{s}$, is defined by means of the normal to the slip plane, $\underline{\mathbf{n}}^{s}$, and the slip direction, $\underline{\mathbf{l}}^{s}$.

$$
\underline{\sim}^{s}=\left(\underline{\mathbf{n}}^{s} \otimes \underline{\mathbf{l}}^{s}+\underline{\mathbf{l}}^{s} \otimes \underline{\mathbf{n}}^{s}\right) / 2
$$

It is used to compute the resolved stress, and also for the evaluation of the plastic strain rate tensor, in a small strain framework:

$$
\tau^{s}=\mathbf{m}_{\sim}^{s}: \underset{\sim}{\sigma} \quad{\underset{\sim}{\dot{\varepsilon}^{p}}}^{p}=\sum_{s} \dot{\gamma}^{s} \mathbf{m}^{s}
$$

Evaluation of the scale transition by finite element analysis (FEA). A polycrystal computation is performed on 200-grain periodic aggregates (Fig.2). The elements used are quadratic tetrahedra that respect grain boundaries. FEA is performed by means of a parallel computation, with the code ZéBuLon, using 12 sub-domains.

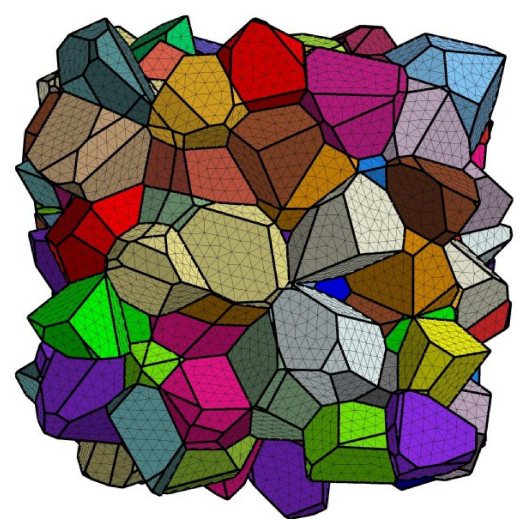

Fig. 2. The periodic 3D mesh used (236312 elements, 332894 nodes). Geometry is composed of Voronoï polyedra. The mesh respects grain boundaries.

The crystallographic orientations randomly assigned to the 200 grains are selected so as to represent the measured texture (Fig.3). Starting from the initial 2016 orientations referenced by the software, and after elimination of the orientations with a too small volume fraction, a tolerance 
cone is associated to each texture vector in order to decrease the number of orientations taken into account.

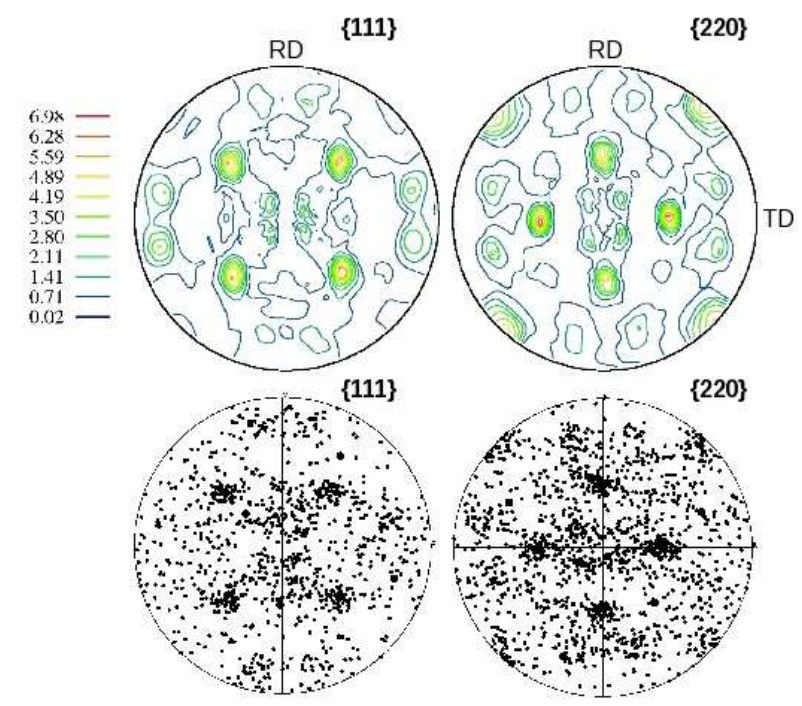

Fig. 3. On the top, texture measured by X-Ray diffraction on a sheet of hot rolled copper OFHC. On the bottom, simplified texture used for finite element computations.

Macroscopic curves obtained by means of FEA are presented in Fig.4.

Mean field scale transition schemes. The total macroscopic strain $\underset{\sim}{\mathbf{E}}$ is classically split into its elastic $\underset{\sim}{\mathbf{E}^{e}}$ and inelastic $\underset{\sim}{\mathbf{E}^{p}}$ parts, while the overall stress $\underset{\sim}{\sum}$ is obtained from :

$$
\left\{\begin{array}{l}
\underset{\sim}{\mathbf{E}}=\underset{\sim}{\mathbf{E}^{e}}+{\underset{\sim}{\mathbf{E}}}^{p} \\
\underset{\sim}{\Sigma}=\underset{\sim}{\mathbf{C}}:\left(\underset{\sim}{\mathbf{E}}-{\underset{\sim}{\mathbf{E}}}^{p}\right),
\end{array}\right.
$$

where $\underset{\sim}{\mathbf{C}}$ is the tensor of the macroscopic elastic moduli. For the sake of simplicity, the individual grains are also supposed to have an isotropic elasticity. Uniform elasticity was used to make sure that the internal stresses come from plasticity only. On the other hand, this assumption is consistent with the description by the chosen uniform field models. This introduces a simplification during elastic loading and at the onset of plastic flow.
In the considered classical elastoviscoplastic scale transition models, the description of the local stress and strain fields is restricted to the set of average stresses $\underset{\sim}{\sigma}$ and strains $\underset{\sim}{\varepsilon}$ over grains having same crystal orientation, the latter being similarly decomposed into their elastic $\underset{\sim}{{\underset{\sim}{e}}^{e}}$ and inelastic ${\underset{\sim}{\varepsilon}}^{p}$ parts with $\underset{\sim}{\sigma}=\underset{\sim}{\mathbf{C}}:\left(\underset{\sim}{\varepsilon}-{\underset{\sim}{\varepsilon}}^{p}\right)$. Classical average relations $<\underset{\sim}{\sigma}>=\sum_{\sim},<\underset{\sim}{\varepsilon}>=\underset{\sim}{\mathbf{E}}$ hold, with in addition $\left\langle\underset{\sim}{\varepsilon^{p}}>={\underset{\sim}{\mathbf{E}}}^{p}\right.$ as a consequence of the homogeneity of the elastic moduli. The same local viscoplastic flow rule as in the FEA is used. It is assumed that it applies also at the scale of the perphase averages to link the evolution of $\underset{\sim}{\varepsilon^{p}}$ to $\underset{\sim}{\sigma}$, ignoring, again for simplicity, the influence of possible intra-phase field fluctuations.

In order to close the set of equations which determine the local stresses from the imposed macroscopic loading history, an additional accomodation equation is required. Following a self-consistent framework, Eshelby-type solutions are used, based on an infinite medium exhibiting the effective properties of the polycrystal, with an embedded inclusion obeying the local constitutive relation of the grains and the shape of which representing the average grain shape. According to Kröner's rule [10], which introduces a purely elastic accomodation, one has :

$$
\underset{\sim}{\sigma}=\underset{\sim}{\Sigma}+\underset{\sim}{\mathbf{C}}:(\underset{\sim}{\mathbf{I}}-\underset{\sim}{\mathbf{S}}):\left({\underset{\sim}{\mathbf{E}}}^{p}-{\underset{\sim}{\varepsilon^{p}}}^{p}\right)
$$

where $\mathbf{S}$ is Eshelby's tensor relative to an ellipsoidal inclusion embedded in an elastic medium with moduli $\underset{\sim}{\mathbf{C}}$. In the present simulations, grains in the polycrystal are equiaxed, consistently with the FEA, and are represented by a sphere. Since inelastic strains are trace free, and assuming uniform isotropic elastic properties, the equation reduces to :

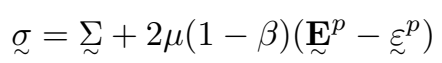

where $\beta=\frac{2(4-5 \nu)}{15(1-\nu)}$ is close to 0.5 with a Poisson's ratio $\nu$ 
close to 0.3 . Since $\mu \gg\|\underset{\sim}{ }\|$, this rules leads to predictions close to Taylor's uniform strain model and overestimates the stress levels.

Improved predictions are obtained when elastoplastic accommodation is taken into account in the accomodation relation, as in Hill's incremental self-consistent model [9] and other schemes derived from it, among which the BerveillerZaoui [3] estimate to which it reduces in the case of spherical inclusions, uniform isotropic elasticity, monotonic and radial loading paths, and when the elastoplastic behaviour of the infinite medium is replaced by a linear isotropic secant approximation, characterised by the secant moduli $\mu^{s e c}=\Sigma^{e q} / 3 E^{e q}$. The accomodation relation reads then :

$$
\begin{aligned}
\underset{\sim}{\sigma}=\underset{\sim}{\sum}+2 \mu(1-\beta) \alpha\left({\underset{\sim}{\mathbf{E}^{p}}}^{\underline{\varepsilon^{p}}}\right) \\
\text { with } \frac{1}{\alpha}=\frac{1}{2}\left(1+\frac{\mu}{\mu^{s e c}}\right)
\end{aligned}
$$

It coincides with Kröner's rule at the beginning of plasticity but leads to softer predictions at higher strains.

In a third model, the $\beta$-rule [15], the accomodation relation is reformulated by means of a phenomenological accommodation variable $\underset{\sim}{\beta}$ which has a non linear evolution with respect to plastic strain. In the case of spherical inclusions and uniform isotropic elasticity, the expression is:

$$
\underset{\sim}{\sigma}=\underset{\sim}{\Sigma}+2 \mu(1-\beta)(\underset{\sim}{\mathbf{B}}-\underset{\sim}{\beta})
$$

with $\underset{\sim}{\mathbf{B}}=<\beta>$

and $\quad \underset{\sim}{\dot{\beta}}=\underset{\sim}{\dot{\varepsilon} p}-D\left(\underset{\sim}{\beta}-\delta{\underset{\sim}{\varepsilon}}^{p}\right)\left\|\dot{\varepsilon}^{p}\right\|$

where $D$ and $\delta$ are not free material parameters. They are constants that are used to calibrate the scale transition rule. They can for instance be adjusted in such a way that the model coincides with Berveiller-Zaoui's approach in the case of a monotonic load. This provides a way to extend this model to more general loading paths, as those considered in the present study; appropriate values are $D=425$ and $\delta=0.035$

Both models (Kröner and $\beta$-rule adjusted on BerveillerZaoui's estimate) were used to simulate the experimental non-proportional tests. The experimental texture is described. The resulting curves are presented in Fig.5 and Fig.6.

\section{Results and comments}

The material parameters used for the single crystal model are given in table 1 . The identification is performed by taking all the tests together, and by minimizing the "distance" between experiment and simulation [4]. The viscosity parameter cannot really be identified here. They were taken in agreement with an other identification realised on dislocation-based single crystal model, but not presented here because of the lack of space. The parameters $R_{0}, Q$, and $b$ are in good agreement with recent result on copper [14]: the initial critical resolved shear stress is relatively low (4 MPa), meanwhile the hardening capacity is rather high $(7 \mathrm{MPa}$ times the numer of active slip systems). A value of 9 for $b$ allows the hardening to be spread on several dozen of cycles, as currently observed in copper[13].

\begin{tabular}{|cccccc|}
\hline$\left.K(\mathrm{s.MPa})^{\frac{1}{n}}\right)$ & $n$ & $R_{0}(\mathrm{MPa})$ & $Q(\mathrm{MPa})$ & $b$ & \\
8 & 20 & 4 & 7 & 9 & \\
\hline$h_{0}$ & $h_{1}$ & $h_{2}$ & $h_{3}$ & $h_{4}$ & $h_{5}$ \\
1 & 1 & 0,2 & 90 & 3 & 2,5 \\
\hline
\end{tabular}

Material parameters of the single crystal model.

The parameters in the interaction matrix correspond respectively to: self hardening $\left(h_{0}\right)$, coplanar interaction $\left(h_{1}\right)$, Hirth lock $\left(h_{2}\right)$, collinear interaction $\left(h_{3}\right)$, glissile junction $\left(h_{4}\right)$, Lomer lock $\left(h_{5}\right)$. Only some of the slip system interactions are active in pure tension, so that all the terms 
cannot be identified using such a type of test. On the other hand, the simulation of the cross hardening tests is really sensitive to both $h_{2}$ and $h_{3}$. The low (0.2) and very high (90) values chosen for these two parameters are mandatory to correctly represent the tension tests $\left(0^{\circ}\right.$ and $\left.45^{\circ}\right)$ after initial shear. In fact, the very high value of $h_{3}$ prevents any collinear slip to occur: this observation is in agreement with values recently obtained by DDD [5].

The set of parameters is used both in FEA (Fig.4) and with the $\beta$-rule (Fig.5). For each case, the simulation is in good agreement with experiment, for the four loading paths. On the other hand, simulations with Kröner's rule (Fig.6) overestimate the macroscopic stress levels, as expected, since the residual stresses are too high in this approach. It is not surprising to observe that the $\beta$-rule is a little better than FEA, in Fig.4. In fact, by definition, the result provided by uniform field models in one simulation involve an equivalent medium around each phase ; as a consequence, the response of this model will be reached by averaging a series of FEA results, where the geometrical distribution of the phases is changed, in order to install a sort of equivalent medium around each grain.

\section{Conclusion and prospects}

The phenomenological crystal plasticity model used in this study [12] provides a rather good agreement between experiments and simulations, made by FEA or by means of an uniform field model ( $\beta$-rule). As expected, Kröner's model over-estimates the macroscopic stress levels.

The most remarkable result deals with the interaction matrix. Following recent DDD computations [5], it has been found that collinear slip is a critical phenomenon, specially in non proportional loadings. A very high value of the related parameter has then been used in the interaction ma- trix, preventing any collinear slip to occur. This choice is mandatory to be able to correctly describe the mechanical response obtained with two orthogonal loading paths.

The result obtained for the matrix interaction coefficients shows that a very high value for collinear slip systems coefficients is necessary to describe the orthogonal loading path. This very high value prevents two collinear slip systems to be activated simultaneously : this observation is in agreement with values recently obtained by discrete dislocation dynamics [5].

\section{Aknowledgement}

D. Caldemaison and T. Chauveau are gratefully acknowledged for their contribution to the experimental part, V. Mounoury and S. Quilici for numerical one.

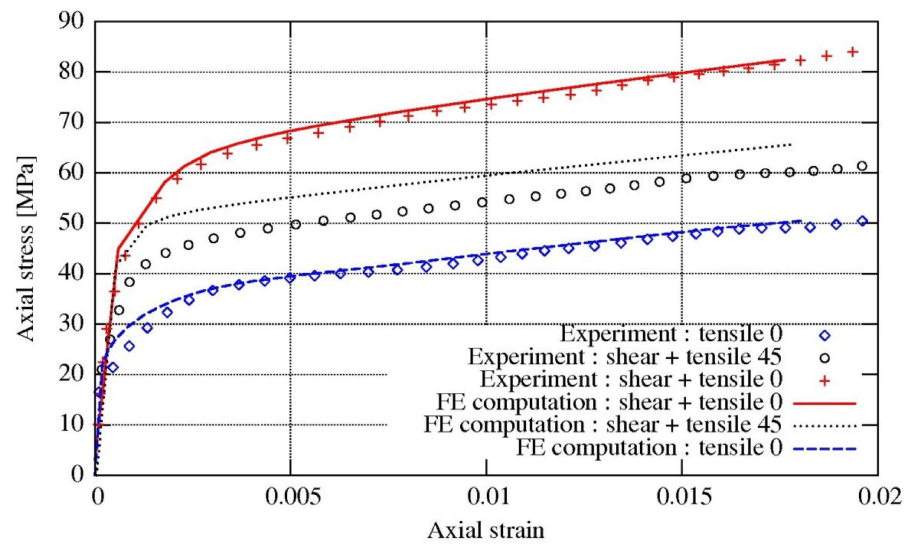

Fig. 4. Macroscopic behaviour simulated by periodic FEA.

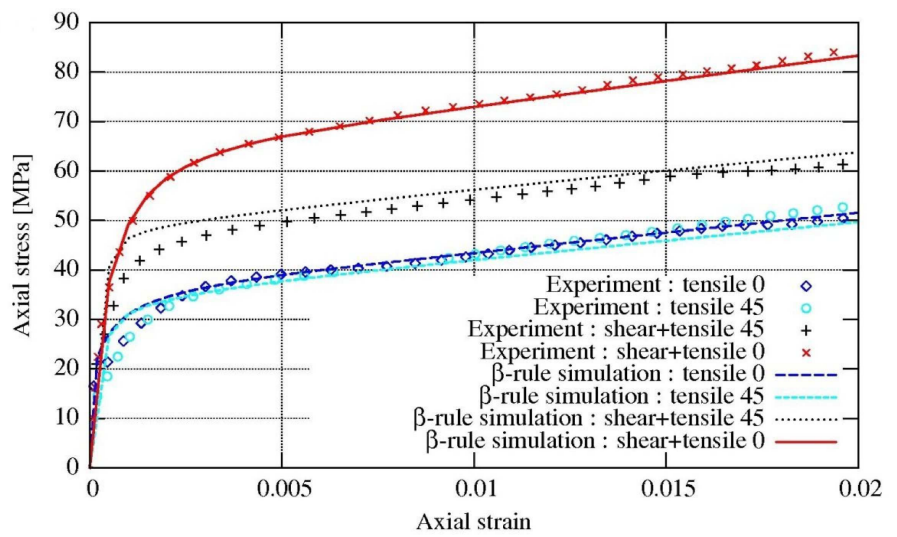

Fig. 5. Macroscopic behaviour modelled with the $\beta$-rule scheme [15]. 


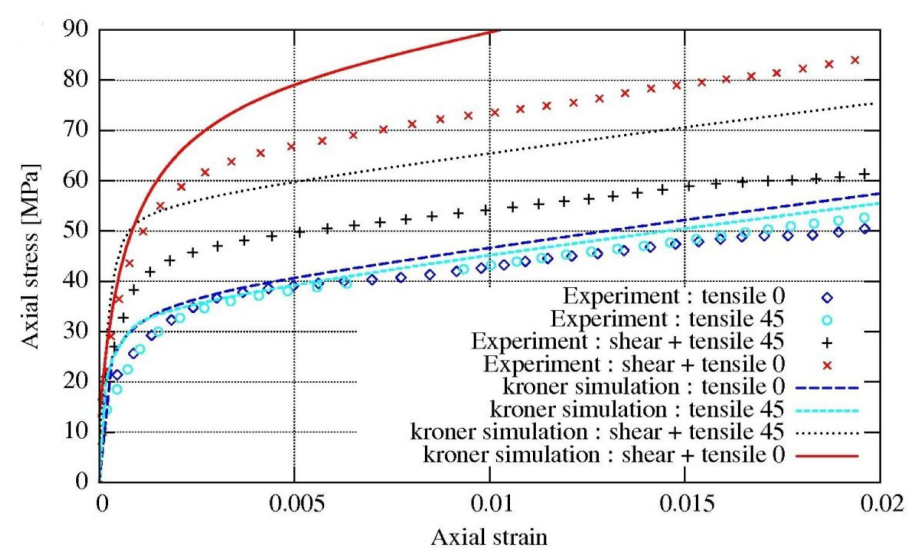

Fig. 6. Macroscopic behaviour modelled with Kröner's model [10].

\section{References}

[1] Asaro R.J., J. Appl. Math. \& Mech., 50 (1983) 921-934

[2] Bacroix B., Genevois P., Teodosiu C., Eur. J. Mech., A/Solids 13(1994) 661-675

[3] Berveiller M., Zaoui A., J. Mech. Phys. Sol. 26 (1979) 325-344

[4] Besson J., Le Riche R., Foerch R., Cailletaud G. Rev. Eur. El. Finis 7 (1998) 567-588

[5] Devincre B., Kubin L., Hoc T., Scripta Mat 54 (2006) 741-746

[6] Franciosi P., Berveiller M., Zaoui A., Acta Metall. 28 (1980) $273-280$

[7] Genevois P., Thèse INPG, Grenoble, France (1992)

[8] Héripré E., Dexet M., Crépin J., Gélébart L., Roos A., Bornert M., Caldemaison D., J. of plasticity 23 (2007) 1512-1539

[9] Hill R., J. Mech. Phys. Sol., 13 (1965), 213-222

[10] Kröner E., Acta Met. 9 (1961) 155-161

[11] Mandel J., Int. J. Solids Structures 1 (1965) 273-295

[12] Méric L., Cailletaud G., J. of Engng. Mat. Technol. 113 (1991) $171-182$

[13] Méric L., Cailletaud G., Gaspérini M. Acta Metall. 42 (1994) $921-935$

[14] Musienko A., Tatschl A., Schmidegg K., Kolednik O., Pippan R., Cailletaud G., Acta Mat. 55 (2007) 4121-4136

[15] Pilvin P., Cailletaud G., Rev. Eur. El. Finis 3 (1994) 515-541

[16] Schmitt J.H., Aernoudt E., Baudelet B., Mat. Sc. and Eng. 75 (1985) 13-20

[17] Tabourot L., Fivel M., Rauch E. Mat. Sc. Eng. A234-236 (1997) 639-642

[18] Wu T.Y., Bassani J., Laird C., C. Proc Roy Soc Lond A (1991) $1-19$ 